DOI 10.4171/JEMS/417

Victor Gerasimov · Leonid Potyagailo

\title{
Quasi-isometric maps and Floyd boundaries of relatively hyperbolic groups
}

Received March 8, 2011 and in revised form March 27, 2012

\begin{abstract}
We describe the kernel of the canonical map from the Floyd boundary of a relatively hyperbolic group to its Bowditch boundary.

Using the Floyd completion we further prove that the property of relative hyperbolicity is invariant under quasi-isometric maps. If a finitely generated group $H$ admits a quasi-isometric map $\varphi$ into a relatively hyperbolic group $G$ then $H$ is itself relatively hyperbolic with respect to a system of subgroups whose image under $\varphi$ is situated within a uniformly bounded distance of the right cosets of the parabolic subgroups of $G$.

We then generalize the latter result to the case when $\varphi$ is an $\alpha$-isometric map for any polynomial distortion function $\alpha$.

As an application of our method we provide in the Appendix a new short proof of a basic theorem of Bowditch characterizing hyperbolicity.
\end{abstract}

Keywords. Floyd boundary, convergence actions, quasi-isometric maps

\section{Introduction}

We study the actions of discrete groups by homeomorphisms of compact Hausdorff spaces such that

(a) the induced action on the space of triples of distinct points is properly discontinuous, and

(b) the induced action on the space of pairs of distinct points is cocompact.

Let $T$ be a compact Hausdorff topological space (a compactum). Denote by $\Theta^{n} T$ the space of subsets of $T$ of cardinality $n$ endowed with the topology induced from the product space.

V. Gerasimov: Department of Mathematics, Federal University of Minas Gerais, Av. Antônio Carlos, 6627, Caixa Postal 702, CEP 30161-970 Belo Horizonte, Brazil; e-mail: victor@mat.ufmg.br, victor.gerasimov@gmail.com

L. Potyagailo: UFR de Mathématiques, Université de Lille 1, 59655 Villeneuve d'Ascq Cedex, France; e-mail: Leonid.Potyagailo@univ-lille1.fr

Mathematics Subject Classification (2010): Primary 20F65, 20F67; Secondary 30F40, 57M07, 22D05 
Recall that an action of a discrete group $G$ by homeomorphisms on $T$ is said to have the convergence property if it satisfies the condition (a) [Bo2], [Tu2]. We also say in this case that the action of $G$ on $T$ is 3-discontinuous.

An action of $G$ on $T$ is called cocompact on pairs or 2-cocompact if $\Theta^{2} T / G$ is compact.

It is shown in [Ge1] that an action with the properties (a), (b) is geometrically finite, that is, every limit point is either conical or bounded parabolic. On the other hand it follows from [Tu3, Theorem 1.C] that every minimal geometrically finite action on a metrizable compactum has the property (b).

An action of $G$ on $T$ is said to be parabolic if it has a unique fixed point. The existence of a nonparabolic geometrically finite action of a finitely generated group $G$ on some compactum $T$ is equivalent to the relative hyperbolicity of $G$ with respect to some collection of proper subgroups [Bo1], [Ya].

W. Floyd $[\mathrm{F}]$ introduced the notion of a boundary of a finitely generated group as follows. The word metric of $G$ is rescaled by a "conformal factor" $f: \mathbb{N} \rightarrow \mathbb{R}_{\geq 0}$ satisfying the conditions (3)-(4) below (we further call $f$ a Floyd function). The Cauchy completion of the resulting metric space is called the Floyd completion and is denoted by $\bar{G}_{f}$. The Floyd boundary is the subspace $\partial_{f} G=\bar{G}_{f} \backslash G$. The action of $G$ on itself by left multiplication extends to a convergence action on $\bar{G}_{f}[\mathrm{Ka}]$.

It is shown in $[\mathrm{F}]$ that for any geometrically finite discrete subgroup $G<\operatorname{Isom~} \mathbb{H}^{3}$ of the isometry group of the hyperbolic space $\mathbb{H}^{3}$, and for the scaling function $f(n)=$ $1 /\left(n^{2}+1\right)(n \in \mathbb{N})$, there exists a continuous $G$-equivariant map $F$ from $\partial_{f} G$ to the limit set $T=\Lambda(G)$. The preimage $F^{-1}(p)$ of a point $p$ is not a single point if and only if $p$ is a parabolic point of rank 1 , in which case it is a pair of points [F]. P. Tukia [Tu1] generalized Floyd's Theorem to geometrically finite discrete subgroups of Isom $\mathbb{H}^{n}$.

If an action of a finitely generated group $G$ on a compactum $T$ has the properties (a) and (b) then by [Ge2] there exists a continuous equivariant map $F_{0}: \partial_{f_{0}} G \rightarrow T$ for the exponential function $f_{0}(n)=\lambda_{0}^{n}$ for some $\left.\lambda_{0} \in\right] 0,1[$. An easy argument then shows (see Corollary 2.8 below) that for any scaling function $f \geq f_{0}$ satisfying $1 \leq f(n) / f(n+1)$ $\leq$ const $(n \in \mathbb{N})$ there also exists a continuous $G$-equivariant map $F: \partial_{f} G \rightarrow T$. The function $f$ is called an (admissible) Floyd function and the map $F$ is the corresponding Floyd map.

Let $\mathrm{Stab}_{G} p$ be the stabilizer of a point $p \in T$ under the action of $G$. If $p$ is parabolic, the subgroup $\operatorname{Stab}_{G} p$ is called the (maximal) parabolic subgroup corresponding to $p$.

For a subset $H$ of $G$ we denote by $\partial H$ its topological boundary in the space $\bar{G}_{f}$. Since the action of $G$ on $\bar{G}_{f}$ has the convergence property, the topological boundary $\partial\left(\operatorname{Stab}_{G} p\right)$ of $\operatorname{Stab}_{G} p$ coincides with its limit set.

Our first result describes the preimage of a point under the map $F$.

Theorem A. Let $G$ be a finitely generated group acting on a compactum $T$ 3-discontinuously and 2-cocompactly. Then for any admissible Floyd function $f$ and for any parabolic point $p \in T$, the corresponding Floyd map $F$ satisfies

$$
F^{-1}(p)=\partial\left(\operatorname{Stab}_{G} p\right) .
$$

Furthermore, if there exist $a \neq b$ such that $F(a)=F(b)=p$, then $p$ is parabolic. 
One of the consequences of Theorem A is that the limit set of a maximal parabolic subgroup for its action on $\bar{G}_{f}$ is a quotient of its Floyd boundary (see Corollary 3.10).

We note that subgroup inclusion does not necessarily imply embedding for the corresponding Floyd boundaries. A trivial example of this type is the inclusion $\mathbb{Z} \hookrightarrow \mathbb{Z}^{2}$ whose extension to the boundaries sends two endpoints of $\mathbb{Z}$ to one endpoint of $\mathbb{Z}^{2}$. Another example can be obtained by considering a Cannon-Thurston type map of a normal hyperbolic subgroup into a hyperbolic group when the point preimages coincide with the boundary of the ending laminations [CaT], [Mi].

Recall that a subgroup $H$ of $G$ is called (strongly) quasiconvex if every quasigeodesic in the Cayley graph of $G$ with endpoints in $H$ lies in a uniform neighborhood of $H$ (depending only on the affine distortion function of the quasigeodesics). Note that the subgroups considered in the above examples are not strongly quasiconvex. We will show below that maximal parabolic subgroups are strongly quasiconvex (Corollary 3.9).

It is proven in [GePo1, Corollary 7.7] that under the above hypotheses on $G$ the Floyd boundary $\partial_{f}\left(\operatorname{Stab}_{G} p\right)$ of $\operatorname{Stab}_{G} p$ injects into $\partial_{f} G$ and coincides with the topological boundary $\partial\left(\operatorname{Stab}_{G} p\right)$. This result and Theorem A imply that

$$
F^{-1}(p)=\partial_{f}\left(\operatorname{Stab}_{G} p\right)
$$

providing a complete description of the Floyd map for relatively hyperbolic groups.

The above discussion leads us to the following:

Questions. Let a finitely generated group $G$ act 3-discontinuously and 2-cocompactly on a compactum $T$. Suppose that $H$ is a strongly quasiconvex subgroup of $G$. Is it true that the group injection $H \hookrightarrow G$ extends to an injection $\partial_{f} H \hookrightarrow \partial_{f} G$ between the Floyd boundaries for some Floyd function $f$ ? In particular is it true that the preimage of the limit set for the action of $H$ on $T$ coincides with the limit set for its action on $\partial_{f} G$ ?

Our next goal is to describe quasi-isometric maps into relatively hyperbolic groups. We have the following:

Theorem B. Let a finitely generated group $G$ act 3-discontinuously and 2-cocompactly on a compactum $T$. Let $\varphi: H \rightarrow G$ be a quasi-isometric map of a finitely generated group $H$ into $G$. Then there exist a compactum $S$, a 3-discontinuous 2-cocompact action of $H$ on $S$, and a continuous map $\varphi_{*}: S \rightarrow T$ such that for every $H$-parabolic point $p \in S$ the point $\varphi_{*} p$ is $G$-parabolic, and $\varphi\left(\operatorname{Stab}_{H} p\right)$ is contained in a uniformly bounded neighborhood of a right coset of $\operatorname{Stab}_{G}\left(\varphi_{*} p\right)$ in $G$.

Using known facts about relative hyperbolicity (see the end of Section 4), Theorem B can be reformulated as follows:

Corollary 1.1. Let $G$ be a finitely generated relatively hyperbolic group with respect to a collection of subgroups $P_{j}(j=1, \ldots, n)$. Let $H$ be a finitely generated group and let $\varphi: H \rightarrow G$ be a quasi-isometric map. Then $H$ is relatively hyperbolic with respect to a collection $Q_{i}$ such that $\varphi$ maps each $Q_{i}$ into a uniform neighborhood of a left coset of some $P_{j}$ (the case $Q_{i}=H$ is allowed). 
Note that several particular cases of Corollary 1.1 are already known: if either the quasiisometric map $\varphi: H \rightarrow G$ is a monomorphism [Hr, Corollary 1.6]; or $\varphi$ admits a quasiisometric inverse map [Dr, Theorem 1.2]; or $G$ is relatively hyperbolic with respect to subgroups which themselves are not relatively hyperbolic with respect to proper subgroups [BDM, Theorem 4.8]. Theorem B provides complete control of the parabolic subgroups of the initial group $H$ under the map $\varphi$ (cf. [BDM, p. 546]). The proof is independent and does not rely on the above results.

We call a scalar nondecreasing function $\alpha: \mathbb{N} \rightarrow \mathbb{R}_{>0}$ a distortion function if $\alpha_{n}=$ $\alpha(n) \geq n$ for all $n \in \mathbb{N}$.

Definition 1.2. A map $\varphi: X \rightarrow Y$ between two graphs $X$ and $Y$ equipped with the standard graph metrics $d_{X}$ and $d_{Y}$ is called $\alpha$-isometric for a distortion function $\alpha$ if

$$
d_{Y}(\varphi(x), \varphi(y)) \leq \alpha\left(d_{x}(x, y)\right) \quad \text { and } \quad d_{X}(x, y) \leq \alpha\left(d_{Y}(\varphi(x), \varphi(y))\right) .
$$

Note the particular cases of $\alpha$-isometric maps: 1) $\alpha(n)=n$ (isometric); 2) $\alpha(n)=c n$ (Lipschitz); 3) $\alpha(n)=c n+c$ (quasi-isometric).

Our next goal is to generalize Theorem B to the case when the distortion function of the map $\varphi$ is polynomial.

Theorem C. Let a finitely generated group $G$ act 3-discontinuously and 2-cocompactly on a compactum $T$. Let $\varphi: H \rightarrow G$ be an $\alpha$-isometric map of a finitely generated group $H$ into $G$ for a polynomial distortion function $\alpha$. Then all statements of Theorem $B$ are true for the group $H$ and the map $\varphi$.

We finish this introduction with a brief description of the methods used in the paper, and outline its content.

One of the crucial points in proving the main results is the fact that the Floyd length of any quasigeodesic in a locally finite connected graph situated far away from the origin is small (the original statement for geodesics in Cayley graphs is due to A. Karlsson [Ka]). In Section 3 we introduce in the (absolute) Cayley graph a system of quasigeodesic convex hulls of subsets of $T$ and horospheres. Using the above property of quasigeodesics with respect to induced Floyd metrics (so called shortcut metrics) on the compactified space $\widetilde{T}=T \sqcup G$, we prove several useful properties of these sets implying Theorem A. The proof of Theorem B is given in Section 4 and is also based on the results of Section 3. In Section 5 we generalize the above approach to the case of $\alpha$-geodesics by proving a generalization of Karlsson's Lemma which is true for any $\alpha$-geodesic once the distortion function $\alpha$ and the Floyd function $f$ form an appropriate pair (see Section 5). We then obtain the proof of Theorem $\mathrm{C}$ following the lines of that of Theorem B. Note that even though Theorem B is formally a particular case of Theorem C, we prefer to keep both statements in the paper. Indeed, the case of quasi-isometric maps is certainly of independent interest. Furthermore historically we first proved Theorem B in the earlier version of our paper [GePo1] and then obtained its generalization.

We would like to stress that the above methods do not require the hyperbolicity of the graph and in particular apply to the (absolute) Cayley graph of a relatively hyperbolic group (and not to the relative one). We hope that this provides a new and useful approach 
to studying convergence groups. To confirm this, we provide in the Appendix a short proof of the fact that the existence of a 3-discontinuous and 3-cocompact action on a compactum without isolated points implies that the group is word-hyperbolic [Bo3].

Note also that the notion of $\alpha$-geodesics is of independent interest. It is proved in [GePo2, Main Theorem] that the property of relative quasiconvexity with respect to $\alpha$-geodesics is equivalent to other definitions of relative quasiconvexity when $\alpha$ is a quadratic polynomial.

\section{Preliminaries}

\subsection{Convergence actions}

Let $T$ be a compactum, that is, a compact Hausdorff space. Let $S^{n} T$ denote the orbit space of the action of the permutation group on $n$ symbols on the product $T^{n}=T \times \stackrel{(n)}{.} \times T$. The elements of $\mathrm{S}^{n} T$ are generalized unordered $n$-tuples. Let $\Theta^{n} T$ denote the open subset of $\mathrm{S}^{n} T$ consisting of all unordered $n$-tuples whose components are distinct. Put $\Delta^{n} T=$ $\mathrm{S}^{n} T \backslash \Theta^{n} T$. So $\Delta^{2} T$ is the image of the diagonal of $T^{2}$.

Convention. Unless otherwise stated, all group actions on compacta are assumed to have the convergence property. We will also assume that $|T|>2$, and so $\Theta^{3} T \neq \emptyset$.

Recall a few common definitions (see e.g. [Bo2], [GM], [Fr], [Tu2]). Let $G$ be a group acting on a compactum $T$. The discontinuity domain $\Omega(G)$ is the set of all points of $T$ where $G$ acts properly discontinuously. The set $\Lambda(G)=T \backslash \Omega(G)$ is the limit set and the points of $\Lambda(G)$ are called limit points. The action of $G$ on $T$ is called minimal if $\Lambda(G)=T$.

It is known that $|\Lambda(G)| \in\{0,1,2, \mathfrak{c}\}$ [Tu2]. The action is called elementary if its limit set is finite.

A point $p \in T$ is called parabolic if $\left|\Lambda\left(\operatorname{Stab}_{G} p\right)\right|=1$.

A limit point $x \in \Lambda(G)$ is called conical if there exists an infinite sequence of distinct elements $g_{n} \in G$ and distinct points $a, b \in T$ such that

$$
\forall y \in T \backslash\{x\}: \quad g_{n}(y) \rightarrow a \in T \wedge g_{n}(x) \rightarrow b .
$$

Denote by Nc $T$ the set of nonconical points of $T$.

A parabolic point $p \in \Lambda(G)$ is called bounded parabolic if the quotient space $(\Lambda(G) \backslash$ $\{p\}) / \mathrm{Stab}_{G} p$ is compact.

The action of $G$ on $T$ is called geometrically finite if every nonconical limit point is bounded parabolic.

A subset $N$ of any set on which $G$ acts is called $G$-finite if $N$ intersects finitely many $G$-orbits.

Lemma 2.1 ([Ge1, Main Theorem]). If the action of $G$ on $T$ is 3-discontinuous and 2-cocompact then:

(a) The set $\mathrm{Nc} T$ is $G$-finite.

(b) For every $p \in \mathrm{Nc} T$ the quotient $(T \backslash\{p\}) / \mathrm{Stab}_{G} p$ is compact. 
It follows from Lemma 2.1 that for a 2-cocompact action of $G$ on $T$ a nonconical point $p \in \mathrm{Nc} T$ is isolated in $T$ if and only if its stabilizer $\operatorname{Stab}_{G} p$ is finite. Hence, a nonconical point with infinite stabilizer is bounded parabolic.

\subsection{Quasigeodesics and Floyd completions of graphs}

Recall that a (c-)quasi-isometric map $\varphi: X \rightarrow Y$ between two metric spaces $X$ and $Y$ is an $\alpha$-isometric map for the affine distortion function $\alpha(n)=c n+c(c>1)$ :

$$
\frac{1}{c} d_{X}(x, y)-c \leq d_{Y}(\varphi(x), \varphi(y)) \leq c d_{X}(x, y)+c,
$$

where $d_{X}, d_{Y}$ are the metrics of $X$ and $Y$ respectively.

Remarks. 1) A quasi-isometric map can in general be multivalued. This more general case can be easily reduced to the case of a single-valued map.

2) Sometimes other terms for quasi-isometric maps are used: large-scale Lipschitz maps [Gr1] or quasi-isometric embeddings [BH] (note that $\varphi$ is not necessarily an injective map).

A path in a graph $\Gamma$ is a distance-nonincreasing map $\gamma: I \rightarrow \Gamma$ from a nonempty convex subset $I$ of $\mathbb{Z}$. The length of the path $\gamma$ is the diameter of $I$ in $\mathbb{Z}$. A subpath is a path which is a restriction of $\gamma$.

A path $\gamma: I \rightarrow \Gamma$ is called a $c$-quasigeodesic if it is a $c$-quasi-isometric map. In the case when $\gamma$ is an isometry, it is called a geodesic.

A ( $c$-quasi-)geodesic path $\gamma: I \rightarrow \Gamma$ defined on a half-infinite interval $I$ of $\mathbb{Z}$ is called a (c-quasi-)geodesic ray; a (quasi-)geodesic path defined on the whole $\mathbb{Z}$ is called a (c-quasi-)geodesic line.

Let $d($,$) denote the canonical shortest path distance function on \Gamma$. We denote by $\mathrm{N}_{D} M$ the $D$-neighborhood of a set $M \subset \Gamma$ with respect to $d$.

We now briefly recall the construction of the Floyd completion of a graph $\Gamma$ due to W. Floyd [F]. Let $\Gamma$ be a locally finite connected graph endowed with a basepoint $v \in \Gamma^{0}$. Let $f: \mathbb{Z}_{>0} \rightarrow \mathbb{R}_{>0}$ be a function satisfying the following conditions:

$$
\begin{aligned}
& \exists K>0 \forall n \in \mathbb{N}: \quad 1 \leq \frac{f(n)}{f(n+1)} \leq K, \\
& \sum_{n \in \mathbb{N}} f(n)<\infty .
\end{aligned}
$$

For convenience we extend the function $f$ to $\mathbb{Z}_{\geq 0}$ by putting $f(0):=f(1)$. We further call any function $f$ satisfying (3)-(4) a Floyd (scaling) function.

Define the Floyd length of an edge joining vertices $x$ and $y$ as $f(n)$ where $n=$ $d(v,\{x, y\})$. Then the Floyd length $L_{f, v}$ (or simply $L_{v}$ for a fixed Floyd scaling function $f$ ) of a path is the sum of the Floyd lengths of its edges. The Floyd distance $\delta_{v}$ (also denoted by $\delta_{f}$ or $\delta_{f, v}$ ) is the shortest path distance:

$$
\delta_{v}(a, b)=\inf _{\alpha} L_{f, v}(\alpha)
$$

where the infimum is taken over all paths between $a$ and $b$. 
It follows from (3) that any two metrics $\delta_{v_{1}}$ and $\delta_{v_{2}}$ are bilipschitz equivalent with Lipschitz constant depending on $d\left(v_{1}, v_{2}\right)$. The Cauchy completion $\bar{\Gamma}_{f}$ of the metric space $\left(\Gamma^{0}, \delta_{v}\right)$ is called the Floyd completion. It is compact and does not depend on the choice of the basepoint $v$. Denote by $\partial_{f} \Gamma$ the set $\bar{\Gamma}_{f} \backslash \Gamma$ and call it the Floyd boundary. The distance $\delta_{v}$ extends naturally to $\bar{\Gamma}_{f}$.

The following lemma shows that the Floyd length of a far quasigeodesic is small.

Lemma 2.2 (Karlsson Lemma). For every $\varepsilon>0$ and every $c>0$, there exists a finite set $D \subset \Gamma$ such that the $\delta_{v}$-length of every c-quasigeodesic $\gamma \subset \Gamma$ that does not meet $D$ is less than $\varepsilon$.

Remark. A. Karlsson [Ka] proved this for geodesics in the Cayley graphs of finitely generated groups. The proof of [Ka] does not use the group action and is still valid for quasigeodesics. In Section 5 we provide a proof of Lemma 2.2 in the more general case of $\alpha$-geodesics (see Lemma 5.1).

Let $S$ be a set of paths of the form $\gamma:[0, n[\rightarrow \Gamma$ of unbounded length starting at the same point $a=\gamma(0)$. Every path $\gamma \in S$ can be considered as an element of the product $\prod_{i \in I} \mathrm{~N}_{i}(a)$ where $\mathrm{N}_{i}(a)$ is the ball in $\Gamma$ centered at $a$ of radius $i$. Since $\Gamma$ is a locally finite graph, the space $\prod_{i \in I} \mathrm{~N}_{i}(a)$ is compact in the Tikhonov topology. So every infinite sequence of paths in $S$ contains a subsequence converging to a path of the form $\delta:[0, \infty[\rightarrow \Gamma$ all of whose initial segments are initial segments of paths in $S$. In particular if $S$ is a set of $c$-quasigeodesics (or more generally $\alpha$-geodesics, see Section 5) then every infinite subset of $S$ admits a subsequence converging to a $c$-quasigeodesic (respectively $\alpha$-geodesic) of infinite length. Note that the infinite limit path exists in a more general context when $\delta(0)$ belongs to a fixed finite set.

Definition 2.3. For a $c$-quasigeodesic ray $r:[0, \infty[\rightarrow \Gamma$ we say that $r$ converges to $a$ point in $\partial_{f} \Gamma$ if $(r(n))_{n}$ is a Cauchy sequence for the $\delta_{f}$-metric. We then also say that $r$ joins the points $r(0)$ and $x=\lim _{n \rightarrow \infty} r(n) \in \partial_{f} \Gamma$.

Proposition 2.4. Let $\Gamma$ be a locally finite connected graph. Then

(a) For each $c>0$ every c-quasigeodesic ray in $\Gamma$ converges to a point in $\partial_{f} \Gamma$.

(b) For every $p \in \partial_{f} \Gamma$ and every $a \in \Gamma$ there exists a geodesic ray joining a and $p$.

(c) Every pair of distinct points in $\partial_{f} \Gamma$ can be joined by a geodesic line.

Proof. (a) Let $r:\left[0, \infty\left[\rightarrow \Gamma\right.\right.$ be a $c$-quasigeodesic ray. Put $x_{n}=r(n)$ and $r_{n}=$ $r\left(\left[n, \infty[)\right.\right.$. For any vertex $v \in \Gamma^{0}$ we have $d(v, r(n)) \rightarrow \infty$. It follows from Karlsson's Lemma that $L_{f, v}\left(r_{n}\right) \rightarrow 0$.

(b) Let $B_{f}(p, R)$ denote the ball in the Floyd metric centered at $p \in \partial_{f} \Gamma$ of radius $R$. For $n \geq 1$, choose $a_{n} \in B_{f}(p, 1 / n)$ and join $a$ to $a_{n}$ by a geodesic segment $\gamma_{n}$. Let $\gamma$ be the limit path for the family $S=\left\{\gamma_{n}: n>0\right\}$. By (a), $\gamma$ converges to a point $q \in \partial_{f} \Gamma$. If $p \neq q$ set $3 \delta=\delta_{1}(p, q)>0$ where $1 \in \Gamma$ is a fixed vertex (we use this notation keeping in mind the case of Cayley graphs). Let $n$ be an integer for which $L_{f, 1}\left(\left.\gamma\right|_{[n, \infty[}\right) \leq \delta$. For $m \geq n$ we can choose $k$ such that $\left.\gamma_{k}\right|_{[0, m]}=\left.\gamma\right|_{[0, m]}$ and 
$\delta_{1}\left(a_{k}, p\right) \leq \delta$. So $L_{f, 1}\left(\left.\gamma_{k}\right|_{[m, k]}\right) \geq \delta$. However the distance $d\left(1, a_{n}\right)$ is unbounded, and by Karlsson's Lemma the quantity $L_{f, 1}\left(\left.\gamma_{k}\right|_{[m, k]}\right)$ should tend to zero. This contradiction shows that $p=q$.

(c) Let $p, q \in \partial_{f} \Gamma$ and $p \neq q$. By (b) there exist geodesic rays $\alpha, \beta:[0, \infty[\rightarrow \Gamma$ such that $\alpha(0)=\beta(0)=a$ and $\alpha(\infty)=p, \beta(\infty)=q$. Let $3 \delta=\delta_{v}(p, q)$. By Karlsson's Lemma every geodesic segment joining a point $\alpha(n)$ in $B_{f}(p, \delta)$ to a point $\beta(n)$ in $B_{f}(q, \delta)$ intersects a finite set $B=B(a, R) \subset \Gamma$. So there exists an infinite sequence of geodesic segments $\gamma_{n}$ passing through a point $b \in B$ whose endpoints converge to the pair $\{p, q\}$. A limit path for such a sequence is the geodesic line in question.

Let $\Gamma_{i}(i=1,2)$ be locally finite connected graphs. The following lemma gives a sufficient condition to extend a quasi-isometric map between the Floyd completions of the graphs.

Lemma 2.5. Let $\varphi: \Gamma_{1} \rightarrow \Gamma_{2}$ be a $c$-quasi-isometric map for some $c \in \mathbb{N}$. Suppose that there exists a constant $D>0$ such that

$$
\frac{f_{2}(n)}{f_{1}(c n)}<D \quad(n \in \mathbb{N}) \text {. }
$$

Then $\varphi$ extends to a Lipschitz map between the Floyd completions $\bar{\Gamma}_{1, f_{1}} \rightarrow \bar{\Gamma}_{2, f_{2}}$.

Proof. To simplify the notations put $\bar{\Gamma}_{i, f_{i}}=\bar{\Gamma}_{i}(i=1,2)$. We denote by $E$ and $K$ the constants from (3) corresponding to $f_{1}$ and $f_{2}$ respectively such that $f_{1}(n) \leq E \cdot f_{1}(n+1)$ and $f_{2}(n) \leq K \cdot f_{2}(n+1)(n \in \mathbb{N})$. By omitting the indices we use the notations $d$ and $\delta$ for the graph distances and the Floyd distances with respect to the functions $f_{i}$ and fixed basepoints denoted by 1 in both graphs $\Gamma_{i}(i=1,2)$.

We first prove that $\varphi$ is a Lipschitz map with respect to the Floyd metrics, i.e.

$$
\forall x, y \in \Gamma_{1}: \quad \delta(x, y) \geq \varepsilon \delta(\varphi(x), \varphi(y))
$$

for some $\varepsilon>0$.

It suffices to prove the statement when $d(x, y)=1$.

By (2) we have $d(\varphi(x), \varphi(y))<c d(x, y)+c=2 c$. Let $\gamma:[0, n] \rightarrow \Gamma_{2}$ be a geodesic realizing the distance $d(\varphi(x), \varphi(y))$, and let $a_{i}=\gamma(i)(i=0, \ldots, n)$ be its vertices where $a_{0}=\varphi(x), a_{n}=\varphi(y)$. We have $\delta(x, y)=f_{1}(d(1,\{x, y\}))$. Assume that $d(1,\{x, y\})=d(1, x)$. Then

$$
\begin{aligned}
d\left(1, a_{i}\right) & \geq d(\varphi(1), \varphi(x))-d\left(\varphi(x), a_{i}\right)-d(1, \varphi(1)) \geq d(\varphi(1), \varphi(x))-2 c-d(1, \varphi(1)) \\
& =d(\varphi(1), \varphi(x))-n_{0},
\end{aligned}
$$

where $n_{0}=2 c+d(1, \varphi(1))$.

Choose $r_{0}>c\left(n_{0}+c\right)$ and let $B\left(1, r_{0}\right)$ denote the ball centered at 1 of radius $r_{0}$. Then for $x \in \Gamma_{1} \backslash B\left(1, r_{0}\right)$ we have $d(\varphi(1), \varphi(x))>n_{0}$. Using the monotonicity of $f_{2}$ and (3) for $x, y \in \Gamma_{1} \backslash B\left(1, r_{0}\right)$ we obtain

$$
\begin{aligned}
\delta(\varphi(x), \varphi(y)) & \leq \sum_{i=0}^{n-1} f_{2}\left(d\left(1,\left\{a_{i}, a_{i+1}\right\}\right)\right) \leq 2 c f_{2}\left(d(\varphi(1), \varphi(x))-n_{0}\right) \\
& \leq 2 c K^{n_{0}} f_{2}(d(\varphi(1), \varphi(x))) .
\end{aligned}
$$


The last term can be estimated using (2), (3) and (6):

$$
\begin{aligned}
f_{2}(d(\varphi(1), \varphi(x))) & \leq D f_{1}(c d(\varphi(1), \varphi(x))) \leq D f_{1}\left(c \cdot d(1, x) / c-c^{2}\right) \\
& \leq D \cdot E^{c^{2}} \cdot f_{1}(d(1, x)),
\end{aligned}
$$

where we used that $d(1, x)-c^{2}>0$. Summing up we conclude

$$
\delta(\varphi(x), \varphi(y)) \leq 2 c D K^{n_{0}} E^{c^{2}} \cdot f_{1}(d(1, x)) .
$$

So (7) is true for the constant $\varepsilon=\left(2 c D K^{n_{0}} E^{c^{2}}\right)^{-1}$ outside of the ball $B\left(1, r_{0}\right)$. By decreasing $\varepsilon$ if necessary, we obtain the inequality (7) everywhere on $\Gamma_{1}$.

The map $\varphi:\left(\Gamma_{1}, \delta\right) \rightarrow\left(\Gamma_{2}, \delta\right)$, being Lipschitz, extends to a Lipschitz map $\bar{\Gamma}_{1} \rightarrow \bar{\Gamma}_{2}$.

Remarks. If, for a function $f$, the value $f(n) / f(2 n)$ is bounded from above (e.g. for any polynomial function), then one can take the same scaling function $f_{1}=f_{2}=f$ for both graphs $\Gamma_{1}$ and $\Gamma_{2}$ independently of $c$.

If the scaling function for the graph $\Gamma_{2}$ is $f_{2}(n)=\alpha^{n}(\alpha \in] 0,1[)$ then to satisfy (6) we can take $f_{1}(n)=\beta^{n}$ as the scaling function for the group $H$ where $\beta=\alpha^{1 / c}$.

Let $G$ be a finitely generated group and let $S$ be a finite generating set for $G$. Denote by $d$ the word metric corresponding to $S$. Let $\bar{G}_{f}$ denote the Floyd completion $G \sqcup \partial_{f} G$ of the Cayley graph of $G$ with respect to $S$ corresponding to a function $f$ satisfying (3)-(4). Condition (3) implies the equicontinuity for the action of every $g \in G$ by left multiplication on $G$ (with an equicontinuity constant depending on $g$ ) [Ka]. So the action of each element $g \in G$ extends by homeomorphism to $\bar{G}_{f}$. Therefore the whole group $G$ acts on $\bar{G}_{f}$ by homeomorphisms. The Floyd metric $\delta_{g}$ is the $g$-shift of $\delta_{1}$ (where 1 is the identity element of $G)$ :

$$
\delta_{g}(x, y)=\delta_{1}\left(g^{-1} x, g^{-1} y\right), \quad x, y \in \bar{G}_{f}, g \in G .
$$

On the space $\bar{G}_{f}$ we also consider the following shortcut pseudometrics.

Definition 2.6. Let $\omega$ be a closed $G$-invariant equivalence relation on $\bar{G}_{f}$. The shortcut pseudometric $\bar{\delta}_{g}$ is the maximal element in the set of symmetric functions $\varrho: \bar{G}_{f} \times \bar{G}_{f}$ $\rightarrow \mathbb{R}_{\geq 0}$ that vanish on $\omega$ and satisfy the triangle inequality, and the inequality $\varrho \leq \delta_{g}$.

For $p, q \in \bar{G}_{f}$ the value $\bar{\delta}_{g}(p, q)$ is the infimum of the finite sums $\sum_{i=1}^{n} \delta_{g}\left(p_{i}, q_{i}\right)$ such that $p=p_{1}, q=q_{n}$ and $\left\langle q_{i}, p_{i+1}\right\rangle \in \omega(i=1, \ldots, n-1)$ [BBI, p. 77]. Obviously, $\bar{\delta}_{g}$ is the $g$-shift of the pseudometric $\bar{\delta}_{1}$. The pseudometrics $\bar{\delta}_{g_{1}}$ and $\bar{\delta}_{g_{2}}$ are bilipschitz equivalent with the same constant as for $\delta_{g_{1}}$ and $\delta_{g_{2}}$.

The pseudometric $\bar{\delta}_{g}$ induces a pseudometric on the quotient space $\bar{G}_{f} / \omega$. We denote this induced pseudometric by the same symbol $\bar{\delta}_{g}$.

The following result will be often used further. 
Lemma 2.7 ([Ge2]). Let $G$ be a finitely generated group acting 3-discontinuously and 2-cocompactly on a compactum $T$ such that $\operatorname{card}(T)>2$. Then there exists $\left.\lambda_{0} \in\right] 0,1[$ and a continuous $G$-equivariant map $F_{0}: \partial_{f_{0}} G \rightarrow T$ where $f_{0}(n)=\lambda_{0}^{n}$.

Summary of the proof of Lemma 2.7. For the reader's convenience we provide a few explanations about the proof of the existence of the Floyd map. We refer the reader to the preprint [Ge2] for more details. It consists of two parts. In the first part it is proven that if a locally compact group $G$ acts 3-properly on a compactum $T$ (i.e. properly on $\Theta^{3} T$ ) and properly and cocompactly on a locally compact Hausdorff space $L$ then there exists a unique topology on $T \sqcup L$ for which the union of these two actions gives rise to a 3-proper action $G \curvearrowright(T \cup L)[\mathrm{Ge} 2,5.2]$.

However to prove the lemma it is enough to consider the particular case when a finitely generated group $G$ acts 3-discontinuously on the Hausdorff space $T$. In this case one can directly show that there exists a locally finite, connected $G$-finite graph $\mathcal{G}$ such that the action of $G$ on the topological space $M=T \cup \mathcal{G}$ is 3-discontinuous [GePo1, Proposition 3.14]. The graph $\mathcal{G}$ is a graph of entourages of $T$ (i.e. neighborhoods of the diagonal $\Delta^{2} T$ of $T^{2}$ ). The properties of its local finiteness and $G$-finiteness are proved in [Ge1].

Since $G$ acts 2-cocompactly on $T$ it is easy to prove that the action $G \curvearrowright \Theta^{2} M$ also admits a compact fundamental set (see e.g. Lemma 3.2 below). Then by [Ge1, Proposition E] there exists a generating entourage $\mathbf{u}$ on $M$ such that the orbit $G \mathbf{u}$ generates the set of entourages Ent $M$ of $M$ as a filter. Then it follows from [Ge2, 6.2] that the system $\left\{\mathbf{u}_{n}=\bigcap S^{n} \mathbf{u} \mid n \in \mathbb{N}\right\}$ is a Frink system of entourages, where $S$ is a finite symmetric generating set of $G$ and $S^{n}$ is the collection of words of $G$ of length at most $n$. By a classical result from general topology the above Frink system gives rise to a Frink metric $\Delta_{\mathbf{u}, S}$ on $M$ which is the maximal among all the metrics $\varrho$ on $M$ satisfying $\left.\varrho\right|_{\mathbf{u}_{n}} \leq 2^{-n}$. Furthermore if $\Delta_{\mathbf{u}, S}(x, y) \leq 2^{-n}$ then $(x, y) \in \mathbf{u}_{n-1}$ [Ke, Lemma 6.2] (or [Ge2, Proposition 6.1.1]). In particular the Frink metric generates the topology of $M$ and $\left\{\mathbf{u}_{n} \mid n \in \mathbb{N}\right\}$ is a countable base for the uniformity of this topology. Let $v \in \mathcal{G}^{0}$ be a basepoint. It is shown in $[\mathrm{Ge} 2,6.3]$ that there exist positive constants $\rho$ and $c$ such that for the exponential Floyd function $f(n)=2^{-n / \rho}$ and for every edge $e \in \mathcal{G}^{1}$ one has

$$
\Delta_{\mathbf{u}, S}(e) \leq c \delta_{f, v}(e)
$$

So (*) implies that the inclusion map $\mathcal{G}^{0} \rightarrow M$ is uniformly continuous. Thus since $M$ is complete, the identity map on $\mathcal{G}$ extends continuously and equivariantly to map $F: \overline{\mathcal{G}}_{f} \rightarrow M$ satisfying

$$
\forall x, y \in \overline{\mathcal{G}}_{f}: \quad \Delta_{\mathbf{u}, S}(F(x), F(y)) \leq c \delta_{f, v}(x, y) .
$$

Since the graph $\mathcal{G}$ is $G$-finite and connected there exists a $G$-equivariant $C$-quasi-isometry $\psi$ between the Cayley graph of $G$ (with respect to some finite generating set) and the graph $\mathcal{G}$. By Lemma 2.5 it extends to a $G$-equivariant map $\psi: \bar{G}_{f_{0}} \rightarrow \overline{\mathcal{G}}_{f}$ where $f_{0}=$ $f^{1 / C}$ and $f(n)=2^{-n / \rho}$ is the above Floyd function for $\mathcal{G}$. So $f_{0}(n)=\lambda_{0}^{n}$ where $\lambda_{0}=$ $2^{-1 / C \rho}$. The map $F_{0}:=F \circ \psi$ satisfies the statement of the lemma. 
Remark. The same proof gives that for any connected locally compact graph $\Gamma$ admitting a cocompact action of the group $G$ there exist an exponential function $f(n)=\mu^{n}(\mu \in$ ]0, 1[) and a $G$-equivariant continuous map $F: \partial_{f} \Gamma \rightarrow T$.

The following corollary shows that every Floyd function $f$ greater than $f_{0}$ admits a Floyd map.

Corollary 2.8. Under the assumptions of Lemma 2.7 for every function $f \geq f_{0}$ satisfying (3)-(4) there exists a continuous $G$-equivariant Floyd map $F: \partial_{f} G \rightarrow T$. In particular this is true for any function $f(n)=1 / \mathcal{P}(n)$ where $\mathcal{P}(n)$ is a polynomial of degree $k>1$ $(n \in \mathbb{N} \cup\{0\}, \mathcal{P}(0) \neq 0)$.

Proof. Let $f_{0}(n)=\lambda_{0}^{n}$ be the function from 2.7. Since $f(n) \leq f_{0}(n)\left(n>n_{0}\right)$ we have $\delta_{f_{0}}(x, y) \leq \delta_{f}(x, y)(x, y \in G)$. So the identity map extends continuously and equivariantly to a map $\chi: \partial_{f} G \rightarrow \partial_{f_{0}} G$. By Lemma 2.7 there exists a continuous $G$ equivariant map $F_{0}: \partial_{f_{0}} G \rightarrow T$. Put $F=F_{0} \circ \chi$.

Since every function of type $1 / \mathcal{P}$ where $\mathcal{P}$ is a polynomial of degree $k>1$ satisfies the conditions (3)-(4) and $f_{0}(n) \leq 1 / \mathcal{P}(n)\left(n>n_{0}\right)$, it admits such a map too.

\section{The orbit compactification space $\widetilde{T}$ and its convex subsets}

In this section we fix a 3-discontinuous and 2-cocompact action by homeomorphisms of a finitely generated group $G$ on a compactum $T$ containing at least three distinct points.

\subsection{The space $\widetilde{T}$}

For a fixed admissible Floyd function $f$ let $F: \partial_{f} G \rightarrow T$ be the Floyd map obtained in Corollary 2.8. We extend $F$ over $\bar{G}_{f}=G \sqcup \partial_{f} G$ to the disjoint union $\hat{T}=T \sqcup G$ by the identity map id : $G \rightarrow G$. We keep the notation $F$ for this extension. The maps $T \stackrel{\text { id }}{\rightarrow} \hat{T} \stackrel{F}{\leftarrow} \bar{G}_{f}$ determine on $\hat{T}$ the pushout topology: a set $S \subset \hat{T}$ is open if and only if $S \cap T$ is open in $T$ and $F^{-1} S$ is open in $\bar{G}_{f}$. The space $\hat{T}$, being the union of two compact spaces $T$ and $F\left(\bar{G}_{f}\right)$, is a compactum.

Since every point $x \in \partial_{f} G$ is the limit of a sequence $g_{n} \in G$ and the map $F$ is equivariant we deduce that $\Lambda(G)=F\left(\partial_{f} G\right)$ for the limit set $\Lambda(G)$ of the action $G \curvearrowright T$. By Lemma 2.1 the set $T \backslash \Lambda(G)$ is $G$-finite. Denote by $\widetilde{T}$ the subspace $\Lambda(G) \sqcup G$ of $\hat{T}$.

Remark. We need to introduce $\hat{T}$ before $\widetilde{T}$ in order to include the exceptional case of 2-ended groups. In this case if the action $G \curvearrowright T$ is not parabolic, $\Lambda(G)$ consists of two points and we need at least one more point to apply Corollary 2.8.

Proposition 3.1. Let $G$ act on compacta $X$ and $Y$ and let $\psi: X \rightarrow Y$ be a $G$-equivariant continuous surjective map. If the action on $X$ is 3-discontinuous, so is the action on $Y$. 
Proof. The map $\psi$ induces a proper $G$-equivariant continuous surjective map $\mathrm{S}^{3} X \rightarrow$ $\mathrm{S}^{3} Y$. Let $K$ and $L$ be compact subsets of $\Theta^{3} Y$. Since $Y$ is Hausdorff, the preimage of every compact set in $\Theta^{3} Y \subset S^{3} Y$ is compact in $\Theta^{3} X$. Thus $K_{1}=\psi^{-1}(K)$ and $L_{1}=$ $\psi^{-1}(L)$ are compact subsets of $\Theta^{3} X$. The action on $X$ is discontinuous so the set $\{g \in G \mid$ $\left.g K_{1} \cap L_{1} \neq \emptyset\right\}$ is finite. By the equivariance of $\psi$ the set $\{g \in G \mid g K \cap L \neq \emptyset\}$ is finite too.

Lemma 3.2. The induced action $G$ on $\widetilde{T}$ is 3-discontinuous and 2-cocompact.

Proof. By [Ka] the group $G$ acts 3-discontinuously on $\bar{G}_{f}=G \sqcup \partial_{f} G$. The Floyd map $F: \bar{G}_{f} \rightarrow \widetilde{T}$ is $G$-equivariant and continuous. By Proposition 3.1 the action on $\widetilde{T}$ is 3-discontinuous.

If $K$ is a compact fundamental set for the action of $G$ on $\Theta^{2}(T)$ then $K_{1}=K \cup\{1\} \times$ $(\widetilde{T} \backslash\{1\})$ is a compact fundamental set for the action of $G$ on $\Theta^{2} \widetilde{T}$.

Remark. The statement of Lemma 3.2 is true for more general 3-proper actions [Ge2] (see also the summary of the proof of Lemma 2.7).

Let $\omega$ be the kernel of the Floyd map $F: \bar{G}_{f} \rightarrow \widetilde{T}$, i.e. $(x, y) \in \omega$ if and only if $F(x)=F(y)$. It determines the shortcut pseudometric $\bar{\delta}_{g}(g \in G)$ on $\bar{G}_{f}$ (see Subsection 2.2). The map $F$ transfers it to a pseudometric on $\widetilde{T}$ also denoted by $\bar{\delta}_{g}$ :

$$
\forall x, y \in \partial_{f} G: \quad \bar{\delta}_{g}(F(x), F(y))=\bar{\delta}_{g}(x, y) .
$$

Since $\bar{\delta}_{g}$ is the maximal pseudometric on $\bar{G}_{f}$ satisfying $\bar{\delta}_{g} \leq \delta_{g}$ for the Floyd metric $\delta_{g}$, by the property $(* *)$ of Section 2 the transferred pseudometric $\bar{\delta}_{g}(g \in G)$ becomes a real metric on $\widetilde{T}$, i.e.

$$
\forall p, q \in \widetilde{T}: \quad \bar{\delta}_{g}(p, q)=0 \Rightarrow p=q .
$$

Any metric $\bar{\delta}_{g}$ determines the topology of $\widetilde{T}$.

Lemma 3.3. Let $H$ be the stabilizer of a parabolic point p. Every $H$-invariant set $M \subset G$ closed in $\widetilde{T} \backslash\{p\}$ is $H$-finite.

Proof. By Lemma 3.2 the action of $G$ on $\widetilde{T}$ is 3-discontinuous and 2-cocompact, so by Lemma 2.1 the space $(\widetilde{T} \backslash\{p\}) / H$ is compact. Since $G \subset \widetilde{T}$ is an orbit of isolated points, the closed subset $M / H$ of $(\widetilde{T} \backslash\{p\}) / H$ consists of isolated points. So the set $M / H$ is finite.

\subsection{Horocycles and horospheres}

By Proposition 2.4(a) every $c$-quasigeodesic ray $\gamma: \mathbb{N} \rightarrow G$ converges to a point $p \in \partial_{f} G$. We call the point $p=\lim _{n \rightarrow \infty} \gamma(n)$ the target of $\gamma$ and denote it by $\gamma(\infty)$. The path $F \circ \gamma$ converges to the point $F(p) \in \widetilde{T}$ which we also call the target. In other words a $c$-quasigeodesic ray extends to a continuous map from $\overline{\mathbb{N}}=\mathbb{N} \cup\{+\infty\}$ to $\widetilde{T}$. The target is necessarily a limit point for the action $G \curvearrowright \widetilde{T}$. So any bi-infinite $c$-quasigeodesic $\gamma: \mathbb{Z} \rightarrow G$ extends to a continuous map of $\overline{\mathbb{Z}}=\mathbb{Z} \cup\{-\infty,+\infty\}$ with $\gamma( \pm \infty) \subset T$. The set $\gamma( \pm \infty)$ is either a pair of limit points or a single limit point.

Every quasigeodesic, either finite or infinite, is defined on a closed subset $J \subset \overline{\mathbb{Z}}$ with $\partial J$ being a pair or a single point. 
Definition 3.4. A bi-infinite $c$-quasigeodesic $\gamma: \mathbb{Z} \rightarrow G$ is called a $c$-horocycle at $p \in T$ if $\gamma(+\infty)=\gamma(-\infty)=p$.

Definition 3.5. The $c$-hull $\mathrm{H}_{c} M$ of a set $M \subset \widetilde{T}$ is the union of $M$ and all $c$-quasigeodesics (finite or infinite) having both endpoints in $M$ :

$\mathrm{H}_{c} M=M \cup\{\gamma(I) \mid \gamma: I \rightarrow G$ is a $c$-quasigeodesic, $I \subset \mathbb{Z}$, and $\gamma(\partial I) \subset M\}$.

The $c$-hull $\mathrm{H}_{c} p$ of a singleton $\{p\} \in T$ is called the $c$-horosphere at $p$.

By $\bar{M}$ we denote the closure of $M$ in $\widetilde{T}$.

Main Lemma. $T \cap \bar{M}=T \cap \overline{\mathrm{H}_{c} M}$ for every $M \subset \widetilde{T}$ and $c>0$.

Proof. Suppose for contradiction that there exist $M$ and $c$ such that $T \cap \overline{\mathrm{H}_{c} M} \backslash \bar{M} \neq \emptyset$. Let $a \in T \cap \overline{\mathrm{H}_{c} M} \backslash \bar{M}$. By Karlsson's Lemma there exists $r>0$ such that the $\delta_{1}$-length of every $c$-quasigeodesic outside of the ball $N_{r}(1) \subset G$ is less than $\varepsilon=\bar{\delta}_{1}(\bar{M}, a) / 2>0$ where $1 \in G$ is a basepoint.

By assumption there exists a $c$-quasigeodesic $\gamma: I=\left[i_{-}, i_{+}\right] \rightarrow G$ such that $0 \in I$, $\gamma(\partial I) \subset M$, and $\gamma(0)$ is arbitrarily close to $a$. So we can assume that $\bar{\delta}_{1}(a, \gamma(0))<\varepsilon$ and $\gamma(0) \notin N_{r_{1}}(1)$ where $r_{1}=r+c r+c / 2$. Let $\gamma_{+}=\left.\gamma\right|_{I \cap \mathbb{N}}$ and $\gamma_{-}=\left.\gamma\right|_{I \cap(-\mathbb{N})}$. For their Floyd lengths $L_{1}$ (see 2.2) we have

$$
\begin{aligned}
L_{1}\left(\gamma_{ \pm}\right) & \geq \bar{\delta}_{1}\left(\gamma\left(i_{ \pm}\right), \gamma(0)\right) \geq \bar{\delta}_{1}\left(\gamma\left(i_{ \pm}\right), a\right)-\bar{\delta}_{1}(a, \gamma(0)) \geq \bar{\delta}_{1}(\bar{M}, a)-\bar{\delta}_{1}(a, \gamma(0)) \\
& \geq 2 \varepsilon-\varepsilon=\varepsilon .
\end{aligned}
$$

So there exists a subsegment $J=\left[j_{-}, j_{+}\right]$of $I$ such that $0 \in J$ and $d\left(1, \gamma\left(j_{ \pm}\right)\right) \leq r$.

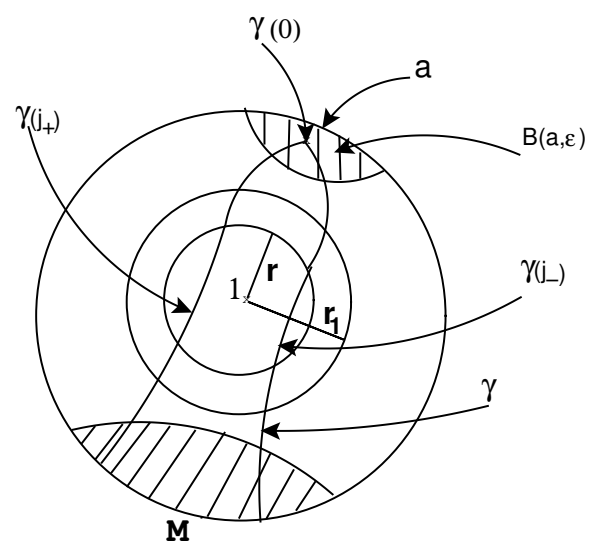

We obtain $d\left(\gamma\left(j_{-}\right), \gamma\left(j_{+}\right)\right)=\operatorname{diam}(\gamma(\partial J)) \leq 2 r$ and length $\left(\left.\gamma\right|_{J}\right) \leq c(2 r+1)$. Thus $d(1, \gamma(0)) \leq d(1, \gamma(\partial J))+d(\gamma(0), \gamma(\partial J)) \leq r_{1}=r+\frac{c}{2}(2 r+1)$. So $\gamma(0) \in N_{r_{1}}(1)$. A contradiction.

We finish the subsection by obtaining several corollaries of the Main Lemma. 
Lemma 3.6. There is no $c$-horocycle at a conical point.

Proof. Let $p \in T$ be a conical point. There exist distinct points $a, q \in T$ and a sequence $\left(g_{n}\right) \subset G$ such that $g_{n}(p) \rightarrow q$ and $g_{n}(x) \rightarrow a$ for all $x \in T \backslash\{p\}$. Suppose for contradiction that $\gamma$ is a $c$-horocycle at $p$.

Let $Q$ be a closed neighborhood of $q$ such that $a \notin Q$. We can assume that $q_{n}=$ $g_{n}(p) \in Q$ for all $n$. So $g_{n}(\gamma(0)) \in \mathrm{H}_{c} Q$. By Lemma 3.2 the action $G \curvearrowright \widetilde{T}$ has the convergence property. So $g_{n}(\gamma(0)) \rightarrow a$. It follows that $a \in\left(\overline{\mathrm{H}_{c} Q} \cap T\right) \backslash(Q \cap T)$, contradicting the Main Lemma.

Until the end of this subsection we fix a parabolic fixed point $p$, and denote by $H$ the stabilizer of $p$ in $G$.

Lemma 3.7. For every $c$ the set $\left(G \cap \mathrm{H}_{c} p\right) / H$ is finite.

Proof. The set $\mathrm{H}_{c} p$ is $H$-invariant. By the Main Lemma it is closed in $\widetilde{T} \backslash\{p\}$ and $p$ is its unique limit point. Thus the set $G \cap \mathrm{H}_{c} p$ is a closed $H$-invariant subset of $\widetilde{T} \backslash\{p\}$. The result follows from Lemma 3.3.

Lemma 3.8. The closure in $\widetilde{T}$ of any $H$-finite subset $M$ of $G$ is $M \cup\{p\}$.

Proof. It suffices to consider the case when $M$ is an $H$-orbit. As $d\left(M, \mathrm{H}_{c} p\right)$ is bounded, the Floyd distance $\delta_{1}\left(m, \mathrm{H}_{c} p\right)$ tends to zero while $m \in M$ tends to $T$.

The following corollary establishes that parabolic subgroups are (strongly) quasiconvex (see the definition in the introduction):

Corollary 3.9. The stabilizer of every parabolic point is strongly quasiconvex.

Proof. Let $\mathrm{H}_{c} p$ be the $c$-horosphere at $p$. By Lemmas 3.7 and 3.8 the set $\mathrm{H}_{c} p \cap G$ is $H$-finite. So there exists $D=D(p, c)>0$ such that for every $h \in H$ we have $d\left(\mathrm{H}_{c} p, h\right) \leq D$. Therefore $H \subset M$ where $M$ is the $D$-neighborhood of $\mathrm{H}_{c} p \cap G$.

We have $\bar{M}=M \cup\{p\}$. By the Main Lemma, $\overline{\mathrm{H}_{c} M}=\mathrm{H}_{c} M \cup\{p\}$. So $\mathrm{H}_{c} M$ is closed in $\widetilde{T} \backslash\{p\}$ and by Lemma 3.3 it is also $H$-finite.

Let $\gamma: I \rightarrow G$ be a $c$-quasigeodesic segment with endpoints in $H \subset M$. Then $\gamma(I)$ and $M$ are both subsets of the $H$-finite set $\mathrm{H}_{c} M$. Hence for any $a \in \gamma(I)$ there exist $h_{i} \in H(i=1,2)$ and $b \in M$ such that $d\left(h_{1}(a), h_{2}(b)\right) \leq$ const. Since $M$ is $H$-invariant we have $h_{1}^{-1} h_{2}(b) \in M$. Then $d(a, M) \leq$ const and so $d(a, H) \leq$ const, where the constant only depends on $c$ and $p$.

\subsection{The point preimage under the Floyd map}

We will now prove the following.

Theorem A. Let $G$ be a finitely generated group acting on a compactum $T$ 3-discontinuously and 2-cocompactly. Then for any admissible Floyd function $f$ and for any parabolic point $p \in T$, the corresponding Floyd map $F$ satisfies

$$
F^{-1}(p)=\partial\left(\operatorname{Stab}_{G} p\right) .
$$

Furthermore, if there exist $a \neq b$ such that $F(a)=F(b)=p$, then $p$ is parabolic. 
Proof. Extending the map $F$ by the identity map as in Subsection 3.1 we can suppose that we have a continuous and $G$-equivariant map $F: \bar{G}_{f} \rightarrow \widetilde{T}$. Denote $H=\operatorname{Stab}_{G} p$. Let $x \in F^{-1}(p)$. We will show that $x \in \partial H \subset \partial_{f} G$. Let $y \in \partial H$. If $y=x$ then there is nothing to prove. If not, then by Proposition 2.4(c) there exists a bi-infinite geodesic $\gamma$ joining $x$ and $y$. It is a horocycle in $\widetilde{T}$, so $\gamma(\mathbb{Z}) \subset \mathrm{H}_{c} p$. By Lemma 3.7, $\gamma(\mathbb{Z})$ is contained in $H g_{1} \cup \cdots \cup H g_{l}$. By Lemma 3.8 the boundary of each $H$-coset is $\{p\}$. So $x=\lim _{n \rightarrow \infty} h_{n} g_{i}$ where $i \in\{1, \ldots, l\}$ and $h_{n} \in H$. Since $d\left(h_{n} g_{i}, h_{n}\right)=d\left(g_{i}, 1\right)$ is constant for all $n$, we have $\delta_{1}\left(x, h_{n}\right) \rightarrow 0$ and $x \in \partial H \subset \partial_{f} G$.

Assume that $a \neq b$. As above, join $a$ and $b$ by a bi-infinite geodesic $\gamma$. Then $\gamma$ is a horocycle in $\widetilde{T}$, and by Lemma 3.6 the point $p=F(a)=F(b)$ is parabolic.

Remark. By Theorem A the preimage of a conical point under an equivariant map is a single point. This is true in a more general setting without assuming that the action of $G$ on $T$ is 2-cocompact [Ge2, Proposition 3.5.2].

Corollary 3.10. In the notation of Theorem $A$ the set $\partial\left(\operatorname{Stab}_{G} p\right)$ is a quotient of the Floyd boundary $\partial_{f_{1}}\left(\operatorname{Stab}_{G} p\right)$ for some Floyd function $f_{1}$.

Proof. Since $H=\mathrm{Stab}_{G} p$ is undistorted in $G$ [Ge1], the inclusion map $H \hookrightarrow G$ is $c$ quasi-isometric for some integer $c$. For a given Floyd function $f$ there exists a Floyd function $f_{1}$ satisfying conditions (3)-(4) such that $f(n) / f_{1}(c n)$ is bounded from above. By Lemma 2.5 the inclusion extends to a continuous map $\bar{H}_{f_{1}} \rightarrow \bar{G}_{f}$. It maps $\partial_{f_{1}}\left(\operatorname{Stab}_{G} p\right)$ onto $\partial\left(\operatorname{Stab}_{G} p\right)$.

Remarks. (a) We confess that we do not understand the argument in [F, p. 216] stating that the preimage under the map $F$ of a parabolic fixed point $p \in \Lambda(G)$ of rank at least two is a single point, where $G<$ Isom $\mathbb{H}^{n}$ is a geometrically finite Kleinian group.

Our statements above complete this argument. Indeed the crucial place was to prove that the preimage $F^{-1}(p)$ of every parabolic point $p \in \partial \mathbb{H}^{n}$ is the limit set of its stabilizer for the action on $\partial_{f} G$. This follows from Theorem A. Then by Karlsson's Lemma the Floyd boundary of a virtually abelian group of rank at least 2 is a point. In particular this is true for any discrete elementary subgroup $H$ of Isom $\mathbb{H}^{n}$ of rank at least 2. By Corollary 3.10 it follows that $F^{-1}(p)$ coincides with the image of the Floyd boundary of $H$, and so is a point. Thus $F^{-1}(p)$ is homeomorphic to the Floyd boundary of $H$.

(b) A stronger statement is proved in [GePo1, Corollary 7.7] where it is shown that the quotient map described in Corollary 3.10 is injective.

\section{Compactification of a quasi-isometric map. Proof of Theorem B}

Let $G$ and $H$ be finitely generated groups with fixed finite generating sets. We denote the corresponding word metrics by the same symbol $d$.

Let $\varphi: H \rightarrow G$ be a $c$-quasi-isometric map. We now choose two Floyd functions $f_{i}$ $(i=1,2)$ satisfying the condition (6) of Lemma 2.5 . Let $\bar{H}_{f_{1}}$ and $\bar{G}_{f_{2}}$ denote the corresponding Floyd completions. 
To simplify notation we put

$$
X=\partial_{f_{2}} G, \quad Y=\partial_{f_{1}} H, \quad \widetilde{X}=X \sqcup G, \quad \widetilde{Y}=Y \sqcup H .
$$

By Lemma 2.5 the map $\varphi_{*}$ extends to a uniformly continuous map $\tilde{Y} \rightarrow \tilde{X}$ which we keep denoting by $\varphi$. By continuity the inequality (7) of the proof of Lemma 2.5 remains valid for this extension.

The kernel $\theta_{0}$ of the composition $\widetilde{Y} \stackrel{\varphi}{\rightarrow} \widetilde{X} \stackrel{F}{\rightarrow} \widetilde{T}$ is a closed equivalence relation on $\tilde{Y}$. We have

$$
(x, y) \in \theta_{0} \Leftrightarrow F \varphi(x)=F \varphi(y) .
$$

The following equivalence relation on $\tilde{Y}$ is closed and $H$-invariant:

$$
\theta=\bigcap\left\{h \theta_{0}: h \in H\right\} .
$$

So $(x, y) \in \theta$ if and only if $(h(x), h(y)) \in \theta_{0}$ for each $h \in H$

Let $\widetilde{S}=\widetilde{Y} / \theta$ and $S=Y / \underset{\sim}{\theta}$. Denote by $\pi$ the quotient map $\widetilde{Y} \rightarrow \widetilde{S}$. It is $H$-equivariant. Since $\theta$ is closed, the space $\widetilde{S}$ is a compactum [Bourb, Prop I.10.8]. The open subspace $A=\pi(H)$ of $\widetilde{S}$ is an $H$-orbit of isolated points. The group $H$ acts 3-discontinuously on $\tilde{Y}$. Since $|\widetilde{S}|>2$, by Proposition 3.1 the action of $H$ on $\widetilde{S}$ is 3-discontinuous too.

The map $\varphi$ sends $\theta$ into the kernel of $F$, and induces a continuous map $\varphi_{*}: \widetilde{S} \rightarrow \widetilde{T}$ such that the following diagram is commutative:

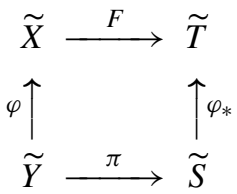

The equivalence $\theta$ determines the shortcut pseudometrics $\bar{\delta}_{g}$ on $\widetilde{S}$ such that

$$
\forall p, q \in \widetilde{Y}, \forall g \in G: \quad \bar{\delta}_{g}(p, q)=\bar{\delta}_{g}([p],[q]) .
$$

Proposition 4.1. The action of $H$ on $\widetilde{S}$ is 2-cocompact.

Proof. By Lemma 3.2 it suffices to verify 2-cocompactness on $S$. Let $[p]$, $[q]$ be distinct $\theta$-classes in $S$. For some $h \in H$ and $p, q \in Y$ we have $\langle h p, h q\rangle \notin \theta_{0}$, so $F \varphi(h p) \neq$ $F \varphi(h q)$. Denote by $K$ a compact fundamental set for the action of $G$ on $\Theta^{2} \widetilde{T}$, i.e.

$$
\Theta^{2} \widetilde{T}=\bigcup\{g K: g \in G\} .
$$

Let $\delta$ be the infimum of the continuous function $\left.\bar{\delta}_{1}\right|_{K}$. It is strictly positive by $\left(8^{\prime}\right)$. There exists $g \in G$ such that $\left\{g^{-1} F \varphi(h p), g^{-1} F \varphi(h q)\right\} \in K$. So $\bar{\delta}_{g}(F \varphi(h p), F \varphi(h q)) \geq \delta$. As $\delta_{g} \geq \bar{\delta}_{g}$ we also have $\delta_{g}(\varphi(h p), \varphi(h q)) \geq \delta$. Let $\gamma$ be a bi-infinite geodesic in $H$ with $\partial \gamma=\{h p, h q\}$. Since $\varphi$ is $c$-quasi-isometric, $\varphi(\gamma)$ is contained in a $c$-quasigeodesic in $G$. So by Karlsson's Lemma there exists $r=r(c, \delta)$ such that $d(g, \varphi(\gamma)) \leq r$. Assume that $d\left(g, g_{0}\right) \leq r$ for $g_{0}=\varphi\left(h_{0}\right), h_{0}=\gamma(0)$. 
By the bilipschitz equivalence of the shortcut metrics, $\bar{\delta}_{g_{0}}(F \varphi(h p), F \varphi(h q) \geq$ $\delta / D(r)$ for a function $D(r)$ depending only on $r$. By $(8), \bar{\delta}_{g_{0}}(\varphi(h p), \varphi(h q))=$ $\bar{\delta}_{g_{0}}(F \varphi(h p), F \varphi(h q))$. By (7) of Lemma 2.5 we obtain

$$
\bar{\delta}_{1}\left(h_{0}^{-1} h p, h_{0}^{-1} h q\right)=\bar{\delta}_{h_{0}}(h p, h q) \geq \varepsilon \bar{\delta}_{g_{0}}(\varphi(h p), \varphi(h q)) \geq \varepsilon \delta / D(r)=\delta_{1} .
$$

Using (11) we conclude that the set $\left\{\left\{s_{1}, s_{2}\right\} \in \Theta^{2} S: \bar{\delta}_{1}\left(s_{1}, s_{2}\right) \geq \delta_{1}\right\}$ is a compact fundamental set for the action of $H$ on $\Theta^{2} S$.

Theorem B. Let a finitely generated group $G$ act 3-discontinuously and 2-cocompactly on a compactum $T$. Let $\varphi: H \rightarrow G$ be a quasi-isometric map of a finitely generated group $H$.

Then there exist a compactum $S$, a 3-discontinuous 2-cocompact action of $H$ on $S$, and a continuous map $\varphi_{*}: S \rightarrow T$ such that for every $H$-parabolic point $p \in S$ the point $\varphi_{*} p$ is $G$-parabolic, and $\varphi\left(\operatorname{Stab}_{H} p\right)$ is contained in a uniformly bounded neighborhood of a right coset of $\operatorname{Stab}_{G}\left(\varphi_{*} p\right)$ in $G$.

Proof. The space $S$ and the map $\varphi_{*}$ are already constructed. We are going to prove that $\varphi_{*}$ maps $H$-parabolic points to $G$-parabolic points. Let $p$ be a parabolic point for the action of $H$ on $\widetilde{S}$ and let $Q$ be its stabilizer. By [Ge1, Main Theorem, d], $Q$ is finitely generated and undistorted in $H$. So the embedding $Q \hookrightarrow H$ is quasi-isometric. Since $Q$ is infinite, there exists a bi-infinite geodesic $\gamma: \mathbb{Z} \rightarrow Q$. Thus $\gamma$ is a $c$-quasigeodesic in $H$ for some $c>0$.

By Proposition 4.1 the action of $H$ on $\widetilde{S}$ is 2-cocompact. By the Main Lemma the boundary of $Q$ in $\widetilde{S}$ is $\{p\}$. In particular, $\gamma$ is a $c$-horocycle. Since $\varphi$ is quasi-isometric, the path $\varphi \circ \gamma: \mathbb{Z} \rightarrow \widetilde{T}$ is an $l$-quasigeodesic for some constant $l>0$. The continuity of $\varphi_{*}$ and the commutativity of the above diagram imply that $\lim _{n \rightarrow \pm \infty} \varphi_{*}(\gamma(n))=\varphi_{*}(p)$. Thus $\varphi_{*} \circ \gamma$ is an $l$-horocycle at the point $\varphi_{*} p$. It follows from Lemma 3.6 that $\varphi_{*} p$ is parabolic for the action of $G$ on $T$.

Every $h \in Q$ belongs to a bi-infinite geodesic in $Q$. So by the above argument we have $\varphi(Q) \subset \mathrm{H}_{l}\left(\varphi_{*} p\right)$. Since $\mathrm{Nc} T$ is $G$-finite there exists a finite set $W$ of $G$ nonequivalent parabolic points of $G$ such that $g\left(\varphi_{*} p\right)=q \in W$ for some $g \in G$. Thus $g\left(\mathrm{H}_{l}\left(\varphi_{*} p\right)\right)=\mathrm{H}_{l} q$. By Lemma 3.7 the set $G \cap \mathrm{H}_{l} q$ is $\operatorname{Stab}_{G} q$-finite. So there exists a uniform constant $C>0$ such that for all $q \in W$ and all $y \in \mathrm{H}_{l} q, d\left(y, \operatorname{Stab}_{G} q\right) \leq C$. So $d\left(g \varphi(Q), \operatorname{Stab}_{G} q\right)=d\left(\varphi(Q), g^{-1} \operatorname{Stab}_{G} q\right)=d\left(\varphi(Q), \operatorname{Stab}_{G}\left(\varphi_{*} p\right) g^{-1}\right) \leq C$.

Proof of Corollary 1.1. Suppose that $G$ is a finitely generated relatively hyperbolic group with respect to parabolic subgroups $P_{i}(i=1, \ldots, n)$ in the strong sense of Farb [Fa]. Then by [Bo1] (see also [Hr]) the group $G$ has a geometrically finite 3 -discontinuous action on a compact metrizable space $X$. It follows from [Tu3, Theorem 1.C] that the space $\Theta^{2} X / G$ is compact. Let $S$ be a compactum as in Theorem B on which the group $H$ acts 3 -discontinuously and 2-cocompactly. By [Ge1] this action is geometrically finite, the set of parabolic points is $H$-finite, and their stabilizers are all finitely generated. It follows from [Ya] that $H$ is relatively hyperbolic with respect to the stabilizers $Q_{i}(i=1, \ldots, k)$ of $H$-nonequivalent parabolic points. By Theorem B the image of every parabolic sub- 
group of $H$ by $\varphi$ is contained in a uniform neighborhood of a right coset of the corresponding parabolic subgroup of $G$ which is a left coset of one of $P_{j}(j=1, \ldots, n)$. The corollary follows.

\section{5. $\alpha$-isometric rigidity. Proof of Theorem C}

The goal of this section is to generalize Theorem B to the case of $\alpha$-isometric maps (see (1)).

Theorem C. Let a finitely generated group $G$ act 3-discontinuously and 2-cocompactly on a compactum $T$. Let $\varphi: H \rightarrow G$ be an $\alpha$-isometric map of a finitely generated group $H$ into $G$ for a polynomial distortion function $\alpha$. Then all statements of Theorem $B$ are true for the group $H$ and the map $\varphi$.

The proof will follow the lines of the proof of Theorem B using a few new facts.

Definition. A path $\gamma: I \rightarrow \Gamma$ in a graph $\Gamma$ is called an $\alpha$-geodesic if the map $\gamma$ is $\alpha$-isometric.

We start with the following generalization of Karlsson's Lemma 2.2:

Lemma 5.1 (Generalized Karlsson Lemma). Let $\Gamma$ be a locally finite connected graph endowed with a basepoint $v \in \Gamma^{0}$. Let $\alpha: \mathbb{N} \rightarrow \mathbb{R}_{>0}$ and $f: \mathbb{N} \rightarrow \mathbb{R}_{>0}$ be respectively the distortion and Floyd scaling function satisfying

$$
\sum_{n \in \mathbb{N}} \alpha_{2 n+1} f_{n}<\infty
$$

where $\alpha_{n}=\alpha(n)$ and $f_{n}=f(n)$. Then for every $\varepsilon>0$ there exists $r=r(\varepsilon)$ such that for every $\alpha$-distorted path $\gamma: I \rightarrow \Gamma$ the condition $d(v, \gamma)>r$ implies that $L_{f, v}(\gamma)<\varepsilon$ for the Floyd length $L_{f, v}$ of $\gamma$.

Proof. Let $0 \in I$ realize the distance $d(v, \gamma)=d(v, \gamma(0))>r$. For every $i>r$ we put $x_{i}=\min \{x \in I \mid \forall t \geq x: d(\gamma(t), v) \geq i\}$. We have $d\left(v, \gamma\left(x_{i}\right)\right)=i$. Indeed, if not then $d\left(v, \gamma\left(x_{i}\right)\right)>i$ and by the triangle inequality $d\left(v, \gamma\left(x_{i}-1\right)\right)>i-1$ and so $d\left(v, \gamma\left(x_{i}-1\right)\right) \geq i$, which is impossible by the choice of $x_{i}$. So the interval $I$ is subdivided into the intervals $I_{i}=\left[x_{i}, x_{i+1}\right.$ [ such that for all $t \in I_{i}, d(v, \gamma(t)) \geq$ $d\left(v, \gamma\left(x_{i}\right)\right)=i$. By the triangle inequality $d\left(\gamma\left(x_{i}\right), \gamma\left(x_{i+1}\right)\right) \leq 2 i+1$. Since $\gamma$ is an $\alpha$ geodesic we have $x_{i+1}-x_{i} \leq \alpha_{2 i+1}$. For the Floyd length this yields $L_{\delta_{f, v}}\left(\left.\gamma\right|_{\left[x_{i}, x_{i+1}\right]}\right) \leq$ $f\left(d\left(v, \gamma\left(I_{i}\right)\right)\right) \cdot$ length $_{d} \gamma\left(I_{i}\right)=\alpha_{2 i+1} f_{i}$. Thus

$$
L_{f, v}(\gamma) \leq \sum_{i=r}^{k} \alpha_{2 i+1} f_{i}+\alpha_{2 k} f_{k},
$$

where $k<\infty$ only if $I$ is finite and $d\left(v, \gamma\left(x_{k}\right)\right)=d(v, \gamma(\max I))$. By (12) there exists $r=r(\varepsilon)$ such that $\sum_{i=r}^{k} \alpha_{2 i+1} f_{i}<\varepsilon / 2$ and $\alpha_{2 k} f_{k} \leq \alpha_{2 k+1} f_{k}<\varepsilon / 2$ as $\left(\alpha_{n}\right)_{n}$ is a nondecreasing sequence. The lemma is proved. 
Definition 5.2. We call the couple $(f, \alpha)$ of a Floyd scaling function $f$ and a distortion function $\alpha$ appropriate if it satisfies (12).

Corollary 5.3. If the pair $(f, \alpha)$ is appropriate then every $\alpha$-geodesic ray in a locally finite connected graph $\Gamma$ converges to a point in $\partial_{f} \Gamma$.

Proof. One repeats the argument of Proposition 2.4 where instead of the Karlsson Lemma one uses the Generalized Karlsson Lemma.

The following result generalizes Lemma 2.5 to the case of $\alpha$-isometric maps:

Lemma 5.4. Let $\Gamma_{i}(i=1,2)$ be locally finite connected graphs and $\varphi: \Gamma_{1} \rightarrow \Gamma_{2}$ be an $\alpha$-isometric map for some distortion function $\alpha: \mathbb{N} \rightarrow \mathbb{N}$. Suppose that the Floyd scaling functions $f_{i}: \mathbb{N} \rightarrow \mathbb{R}_{>0}(i=1,2)$ satisfy

$$
f_{2}(n) / f_{1}\left(\alpha_{n}\right) \leq D \quad(n \in \mathbb{N})
$$

for some constant $D>0$. Then $\varphi$ extends to a Lipschitz map (denoted by the same letter) $\varphi: \bar{\Gamma}_{1, f_{1}} \rightarrow \bar{\Gamma}_{2, f_{2}}$.

Proof. We keep the notations of the proof of Lemma 2.5. Using the $\alpha$-isometric map $\varphi$ the same argument of Lemma 2.5 shows that for all $x, y \in \Gamma_{1} \backslash B\left(1, r_{0}\right)$ such that $d(x, y)=1$ and $r_{0}=\alpha\left(n_{0}\right), n_{0}=\alpha_{1}+d(1, \varphi(1))$ the following is true:

$$
\begin{aligned}
d(\varphi(x), \varphi(y) & <\alpha_{1}, \\
\delta_{f_{2}}(\varphi(x), \varphi(y)) & \leq \alpha_{1} K^{n_{0}} f_{2}\left(d(\varphi(1), \varphi(x)) \leq \alpha_{1} K^{n_{0}} D f_{1}(\alpha(d(\varphi(1), \varphi(x)))\right. \\
& \leq \alpha_{1} K^{n_{0}} D f_{1}(d(1, x)) .
\end{aligned}
$$

So for all $x, y \in \Gamma_{1}$ we obtain $\delta_{f_{2}}(\varphi(x), \varphi(y)) \leq$ const $\cdot \delta_{f_{1}}(x, y)$. Thus $\varphi$ extends to a Lipschitz map $\bar{\Gamma}_{1, f_{1}} \rightarrow \bar{\Gamma}_{2, f_{2}}$.

Remark. For the Floyd scaling function $f_{1}=1 / \mathcal{P}_{1}$ and for the distortion function $\alpha=\mathcal{P}_{2}$ where $\mathcal{P}_{i}$ are polynomials of degree $>1$ we put $f_{2}=f_{1} \circ \alpha=1 / \mathcal{P}_{1} \circ \mathcal{P}_{2}$. Then the condition (13) is satisfied.

For a distortion function $\alpha$ we introduce similarly to 3.5 the $\alpha$-hull $\mathrm{H}_{\alpha} M$ of a set $M$ :

$$
\mathrm{H}_{\alpha} M=M \cup\{\gamma(I) \mid \gamma: I \rightarrow G \text { is } \alpha \text {-geodesic, } I \subset \mathbb{Z} \text {, and } \gamma(\partial I) \subset M\} \text {. }
$$

We have the following generalization of the Main Lemma:

Lemma 5.5 (Main Lemma for $\alpha$-hulls). For every polynomial function $\alpha: \mathbb{N} \rightarrow \mathbb{R}_{\geq 0}$,

$$
\forall M \subset \widetilde{T}: \quad T \cap \bar{M}=T \cap \overline{\mathrm{H}_{\alpha} M} .
$$


Proof. Fix a Floyd function $f=1 / \mathcal{P}$ such that $\mathcal{P}$ is a polynomial of degree $>1$ and the pair $(f, \alpha)$ is appropriate. By Corollary 2.8 for the function $f$ there exists a Floyd map $F: \bar{G}_{f} \rightarrow \widetilde{T}$. It gives the shortcut metric $\bar{\delta}_{1, f}$ on $\widetilde{T}$ satisfying $\delta_{1, f} \geq \bar{\delta}_{1, f}$ (see (8) and $\left.\left(8^{\prime}\right)\right)$.

Using the Generalized Karlsson Lemma (instead of Karlsson's Lemma 2.2) in the argument of the Main Lemma we find that the Floyd length of every $\alpha$-geodesic outside of the ball of a radius $r$ is less than $\varepsilon=\bar{\delta}_{1, f}(\bar{M}, a) / 2>0$. By the same argument, every $\alpha$-geodesic with endpoints in $M$ lies in the union of $\bar{M}$ and a ball of finite radius centered at $1 \in G$. The lemma follows.

Definition. A bi-infinite $\alpha$-geodesic $\gamma: \mathbb{Z} \rightarrow G$ is called an $\alpha$-horocycle at a point $p \in T$ if $\gamma(+\infty)=\gamma(-\infty)=p$.

Using the Generalized Karlsson Lemma and Lemma 5.5 (instead of 2.2 and Main Lemma) in the proofs of Lemmas 3.7 and 3.6 we obtain the following generalizations for $\alpha$-geodesics.

Lemma 5.6. For every polynomial distortion function $\alpha$ there is no $\alpha$-horocycle at a conical point.

Lemma 5.7. For every polynomial distortion function $\alpha$ the set $\left(G \cap \mathrm{H}_{\alpha} p\right) / H$ is finite.

Proof of Theorem $C$. Let $\varphi: H \rightarrow G$ be an $\alpha$-isometric map. Following the above Remark we fix Floyd scaling functions $f_{1}$ and $f_{2}$ of polynomial type such that $f_{1}=1 / \mathcal{P}_{1}$ and $f_{2}=1 / \mathcal{P}_{1} \circ \alpha\left(\operatorname{deg} \mathcal{P}_{1}>1\right)$. As before we put

$$
X=\partial_{f_{2}} G, \quad Y=\partial_{f_{1}} H, \quad \tilde{X}=X \sqcup G, \quad \tilde{Y}=Y \sqcup H .
$$

For the Floyd function $f_{2}$ by Corollary 2.8 there exists a Floyd map $F: \widetilde{X} \rightarrow \widetilde{T}$.

As in the proof of Theorem B we define the quotients $\widetilde{S}$ and $S$ of the spaces $\tilde{Y}$ and $Y$ respectively. The action $H \curvearrowright \widetilde{S}$ is 3-discontinuous. To show that it is 2-cocompact we modify the proof of Proposition 4.1 as follows. For a geodesic $\gamma \subset H$ we obtain the $\alpha$-geodesic $\varphi(\gamma) \subset G$.

The series $\Sigma=\sum_{k} \alpha_{2 k+1} f_{2, k}$ converges. As $\alpha$ is a polynomial function we have

$$
\frac{\alpha(2 k+1)}{\mathcal{P}_{1}(\alpha(k))} \leq \text { const } \cdot \frac{\alpha(k)}{\mathcal{P}_{1}(\alpha(k))} .
$$

Since $\operatorname{deg} \mathcal{P}_{1}>1$ the series $\sum_{k} \alpha(k) / \mathcal{P}_{1}(\alpha(k))$ converges and so does $\Sigma$. Thus applying the Generalized Karlsson Lemma to the space $\widetilde{X}$, we conclude that $\varphi(\gamma)$ is within a distance $r=r(\alpha, \delta)$ of the basepoint $g$. So $d\left(g, g_{0}\right) \leq r$ where $g_{0}=\varphi\left(h_{0}\right)$ for some $h_{0} \in H$. Applying now Lemma 5.4 (instead of 2.5) we see that the action $H \curvearrowright \Theta^{2} S$ admits a compact fundamental set.

To show that $\varphi_{*}(p) \in T$ is parabolic for a parabolic point $p \in S$, we proceed similarly. Since every parabolic subgroup is undistorted in $H$, there exists a $c$-quasigeodesic horocycle $\gamma \subset H$ at the limit point $p$. The composition $\varphi \circ \gamma$ is an $\alpha$-geodesic horocycle 
in $\widetilde{T}$. It follows from Lemma 5.6 that its unique limit point $\varphi_{*}(p) \in T$ is parabolic for the action $G \curvearrowright T$.

Similarly applying Lemma 5.7 we deduce that the set $G \cap \mathrm{H}_{\alpha} q$ is $\operatorname{Stab}_{G} q$-finite. This implies that the image of every parabolic subgroup $Q$ of $H$ is within a uniformly bounded distance of a right coset of $\operatorname{Stab}_{G}\left(\varphi_{*} p\right)$ in $G$. Theorem C follows.

\section{Appendix: a short proof that 3-cocompactness of an action implies word-hyperbolicity of the group}

As an application of our method we give a short proof of the following theorem of B. Bowditch:

Theorem ([Bo3]). Let $G$ be a finitely generated group acting 3-discontinuously and 3cocompactly on a compactum $T$ without isolated points. Then $G$ is word-hyperbolic.

We start with the following.

Lemma 6.1. Let a group $G$ act 3-discontinuously on a compactum $T$. Let $p, q$ be distinct nonconical points in $T$ and let $K$ be a compact subset of $T^{2} \backslash \Delta^{2} T$. Then the set $S=$ $\{g \in G:(g p, g q) \in K\}$ is finite.

Proof. We will use some simple facts about convergence actions on compacta proved in [Ge1]. Assume that $S$ is infinite. The compact $K$ can be covered by finitely many closed subproducts of the form $A \times B$ with $A \cap B=\emptyset$. So we can assume that $K=A \times B$ where $A, B$ are closed disjoint sets. The set $\Lambda_{\text {rep }} S$ of repellers of limit crosses for $S$ (see [Ge1, Subsection 18]) is nonempty. It is contained in $\{p, q\}$ since otherwise, for some $g \in S$, the pair $\{g p, g q\}$ becomes arbitrarily small.

So $S$ contains an infinite subset $S_{1}$ with $\Lambda_{\text {rep }} S_{1}$ being a single point. Without loss of generality we can assume that $S=S_{1}$ and $\Lambda_{\text {rep }} S=\{p\}$. The set $\Lambda_{\text {attr }} S$ of attractors of limit crosses is contained in $B$. Let $B_{1}$ be a closed neighborhood of $B$ disjoint from $A$. Thus, for $a \in T \backslash\{p\}$, the set $\left\{g \in S: g a \notin B_{1}\right\}$ is finite since it has no limit crosses. Hence $\{\{g p, g a\}: g \in S\}$ is contained in a compact subset of $\Theta^{2} T$. So $p$ is conical by [Ge1, Definition 3].

Remark. Note that with the additional assumption that $T$ is metrizable the above lemma easily follows from Gehring-Martin's definition of the convergence property.

Corollary. If $G$ acts 3-discontinuously and 3-cocompactly on a compactum $T$ without isolated points then every point of $T$ is conical.

Proof. Clearly, 3-cocompactness implies 2-cocompactness, hence every nonconical point is either isolated or parabolic [Ge1]. By assumption the discontinuity domain is empty. Assume that parabolic points do exist. Hence there exist at least two parabolic points since otherwise we must have a discontinuity domain.

Let $p, q$ be distinct parabolic points and let $L$ be a compact fundamental set for the action of $G$ on $\Theta^{3} T$. We can assume that $L$ has the form $\bigcup_{i=1}^{n} A_{i} \times B_{i} \times C_{i}$ where 
$A_{i}, B_{i}, C_{i}$ are closed disjoint subsets of $T$. For every $a \in T \backslash\{p, q\}$ there exist $g_{a} \in G$ such that $g_{a}(p, q, a) \in L$. By Lemma 6.1 the set $\left\{g_{a}: a \in T \backslash\{p, q\}\right\}$ is finite. If $g_{a}(p, q, a) \in A_{i(a)} \times B_{i(a)} \times C_{i(a)}$ then $T \backslash\{p, q\}$ is a union of finitely many closed sets $g_{a}^{-1} C_{i(a)}$. Thus $\{p, q\}$ is open and $p$ and $q$ are isolated, contradicting the assumption.

Proof of the Theorem. Assume that $G$ is not hyperbolic. There exists a sequence of geodesic triangles with sides $\left\{l_{n}, m_{n}, k_{n}\right\}$ so that $d\left(x_{n}, m_{n} \cup k_{n}\right) \rightarrow \infty$ for $x_{n} \in l_{n}$. Using the $G$-action we can make $x_{n}$ equal to 1 for all $n$. By Karlsson's Lemma the Floyd length of $m_{n} \cup k_{n}$ tends to zero and so $\delta_{1}\left(y_{n}, z_{n}\right) \rightarrow 0$ where $\partial l_{n}=\left\{y_{n}, z_{n}\right\}$. Since all $l_{n}$ pass through the same point 1 we can choose a subsequence converging to a geodesic horocycle $l$. By Lemma 3.6 the target of $l$ is not conical, contradicting the above Corollary.

Remark. In [Bo3] the above Theorem is proved without the assumption that $G$ is finitely generated. It is shown in [GePo1, Corollary 3.38] that a group $G$ acting 3-discontinuously and 2-cocompactly on a compactum $T$ is relatively finitely generated with respect to maximal parabolic subgroups. In particular if $G$ acts 3-cocompactly on $T$ by the above Corollary there are no parabolic subgroups and so $G$ is finitely generated.

Acknowledgments. During the work on this paper both authors were partially supported by the ANR grant BLAN 07 - 2183619. We are grateful to the Max-Planck Institut für Mathematik in Bonn, where a part of the work was done. We also thank for the Brasilian-French cooperation grant that supported our work.

The authors are deeply grateful to the referee for her/his useful remarks and corrections.

\section{References}

[BM] Beardon, A., Maskit, B.: Limit sets of Kleinian groups and finite sided fundamental polyhedra. Acta Math. 132, 1-12 (1974) Zbl 0277.30017 MR 0333164

[BDM] Behrstock, J., Druţu, C., Mosher, L.: Thick metric spaces, relative hyperbolicity, and quasi-isometric rigidity. Math. Ann. 344, 543-595 (2009) Zbl 1220.20037 MR 2501302

[Bo1] Bowditch, B. H.: Relatively hyperbolic groups. Int. J. Algebra Comput. 22, no. 3, 1250016, 66 pp. Zbl 1259.20052 MR 2922380

[Bo2] Bowditch, B. H.: Convergence groups and configuration spaces. In: Group Theory Down Under, J. Cossey et al. (eds.), de Gruyter, 23-54 (1999) Zbl 0952.20032 MR 1714838

[Bo3] Bowditch, B. H.: A topological characterisation of hyperbolic groups. J. Amer. Math. Soc. 11, 643-667 (1998) Zbl 0906.20022 MR 1602069

[BH] Bridson, M., Haefliger, A.: Metric Spaces of Non-Positive Curvature. Grundlehren Math. Wiss. 319, Springer, Berlin (1999) Zbl 0988.53001 MR 1744486

[Bourb] Bourbaki, N.: Topologie Générale. Hermann, Paris (1965) MR 0244924

[BBI] Burago, D., Burago, Y., Ivanov, S.: A Course in Metric Geometry, Grad. Stud. in Math. 13, Amer. Math. Soc. (2001) Zbl 0981.51016 MR 1835418

[CaT] Cannon, J., Thurston, W. P.: Group invariant Peano curves. Geom. Topol. 11, 1315-1356 (2007) Zbl 1136.57009 MR 2326947

[Dr] Druţu, C.: Relatively hyperbolic groups: geometry and quasi-isometric invariance. Comment. Math. Helv. 84, 503-546 (2009) Zbl 1175.20032 MR 2507252 
[Fa] Farb, B.: Relatively hyperbolic groups. Geom. Funct. Anal. 8, 810-840 (1998) Zbl 0985.20027 MR 1650094

[F] Floyd, W. J.: Group completions and limit sets of Kleinian groups. Invent. Math. 57, 205-218 (1980) Zbl 0428.20022 MR 0568933

[Fr] Freden, E. M.: Properties of convergence groups and spaces. Conformal Geom. Dynam. 1, 13-23 (1997) Zbl 0983.57029 MR 1452414

[GM] Gehring, F. W., Martin, G. J.: Discrete quasiconformal groups I. Proc. London Math. Soc. 55, 331-358 (1987) Zbl 0628.30027 MR 0896224

[Ge1] Gerasimov, V., Expansive convergence groups are relatively hyperbolic. Geom. Funct. Anal. 19, 137-169 (2009) Zbl 1226.20037 MR 2507221

[Ge2] Gerasimov, V.: Floyd maps for relatively hyperbolic groups. Geom. Funct. Anal. 22, 1361-1399 (2012) Zbl pre06125049 MR 2989436

[GePo1] Gerasimov, V., Potyagailo, L.: Non-finitely generated relatively hyperbolic groups and Floyd quasiconvexity. Groups Geom. Dynam., to appear; arXiv:1008.3470v2 [math.GR] (2010)

[GePo2] Gerasimov, V., Potyagailo, L.: Quasiconvexity in relatively hyperbolic groups. arXiv:1103.1211v1 [math.GR] (2011)

[Gr] Gromov, M.: Hyperbolic groups. In: Essays in Group Theory, S. M. Gersten (ed.), MSRI Publ. 8, Springer, 75-263 (1987) Zbl 0634.20015 MR 0919829

[Gr1] Gromov, M.: Asymptotic invariants of infinite groups. In: Geometric Group Theory II, London Math. Soc. Lecture Note Ser. 182, Cambridge Univ. Press, 1-295 (1993) Zbl 0841.20039 MR 1253544

[Hr] Hruska, G.: Relative hyperbolicity and relative quasiconvexity for countable groups. Algebr. Geom. Topol. 10, 1807-1856 (2010) Zbl 1202.20046 MR 2684983

[KL] Kapovich, M., Leeb, B.: Quasi-isometries preserve the geometric decomposition of Haken manifolds. Invent. Math. 128, 393-416 (1997) Zbl 0866.20033 MR 1440310

[Ka] Karlsson, A.: Free subgroups of groups with non-trivial Floyd boundary. Comm. Algebra, 31, 5361-5376 (2003) Zbl 1036.20032 MR 2005231

[Ke] Kelley, J. L.: General Topology. Grad. Texts in Math. 27, Springer, New York (1975)

[Mi] Mitra, M.: Ending laminations for hyperbolic group extensions. Geom. Funct. Anal. 7, 379-402 (1997) Zbl 0880.57001 MR 1445392

[My] Myrberg, P. J.: Untersuchungen über die automorphen Funktionen beliebig vieler Variablen. Acta Math. 46, 215-336 (1925) JFM 51.0298.02 MR 1555203

[Os] Osin, D.: Relatively hyperbolic groups: intrinsic geometry, algebraic properties and algorithmic problems. Mem. Amer. Math. Soc. 179, no. 843 (2006), vi + 100 pp. Zbl 1093.20025 MR 2182268

[Tu1] Tukia, P.: A remark on the paper by Floyd. In: Holomorphic Functions and Moduli, Vol. II (Berkeley, CA, 1986), MSRI Publ. 11, Springer, New York, 165-172 (1988) Zbl 0653.30027 MR 0955838

[Tu2] Tukia, P.: Convergence groups and Gromov's metric hyperbolic spaces. New Zealand J. Math. 23, 157-187 (1994) Zbl 0855.30036 MR 1313451

[Tu3] Tukia, P.: Conical limit points and uniform convergence groups. J. Reine Angew. Math. 501, 71-98 (1998) Zbl 0909.30034 MR 1637829

[W] Weil, A.: Sur les espaces à structure uniforme et sur la topologie générale. Hermann, Paris (1937) JFM 63.0569.04

[Ya] Yaman, A., A topological characterisation of relatively hyperbolic groups. J. Reine Angew. Math. 566, 41-89 (2004) Zbl 1043.20020 MR 2039323 